EPJ Web of Conferences 60, 17001 (2013)

DOI: $10.1051 /$ epjconf/20136017001

(C) Owned by the authors, published by EDP Sciences, 2013

\title{
Searches for New Physics in Multijet Final States
}

\author{
Carl Vuosalo ${ }^{1, a}$ on behalf of the CMS Collaboration \\ ${ }^{1}$ The Ohio State University
}

\begin{abstract}
A variety of new physics models predict heavy resonances that decay to multiple hadronic jets. These models include axigluons, colorons, diquarks, excited quarks, Randall-Sundrum gravitons, string resonances, and Z' models, among others. Other models make the prediction that high- $p_{T}$ jets will be suppressed, resulting in jet extinction. Using the data collected in 2012 at a center-of-mass energy of $8 \mathrm{TeV}$, the CMS collaboration has made a baseline inclusive jet cross section measurement for comparison with new-physics searches, and then performed searches for jet extinction and resonances that decay to two hadronic jets. The results of these searches will be presented. No evidence of new physics has been observed, and these results set new limits on the parameters of these models.
\end{abstract}

\section{Introduction}

A major goal of the Compact Muon Solenoid (CMS) experiment [1] at the Large Hadron Collider (LHC) is the discovery of new physics (NP) beyond the standard model (SM). Many NP theoretical models predict jet extinction or production of resonances that decay to paired jets. During 2012, the CMS detector recorded $19.6 \mathrm{fb}^{-1}$ of pp data at a center-of-mass energy of $8 \mathrm{TeV}$. Several CMS analyses have exploited this rich data set to make a measurement of the inclusive jet cross section and to search for these NP signals.

\section{Measurement of Differential Inclusive Jet Cross Sections}

The inclusive jet cross section provides an important baseline for comparison with new-physics models. Additionally, the measurement provides a method of assessment of Quantum Chromodynamic (QCD) calculation frameworks and parton distribution functions (PDFs) used with those frameworks. CMS has performed the measurement of the double-differential inclusive jet cross section in terms of $p_{T}$ and rapidity by using $10.7 \mathrm{fb}^{-1}$ of the $20128 \mathrm{TeV} \mathrm{pp}$ dataset [2]. The measurement uses six, staggered triggers to cover a $p_{T}$ range from $74-2500 \mathrm{GeV}$ and a range of parton momentum fraction $x$ from $0.019-0.625$. The data are corrected for detector smearing effects and jet energy resolution. The dominant systematic uncertainty for data comes from the jet energy scale and ranges from $15-40 \%$.

The theory prediction is calculated at next-to-leadingorder (NLO) by NLOJet++ $[3,4]$ in the fastNLO framework [5]. Its dominant systematic uncertainty comes

\footnotetext{
ae-mail: cvuosalo@cern.ch
}

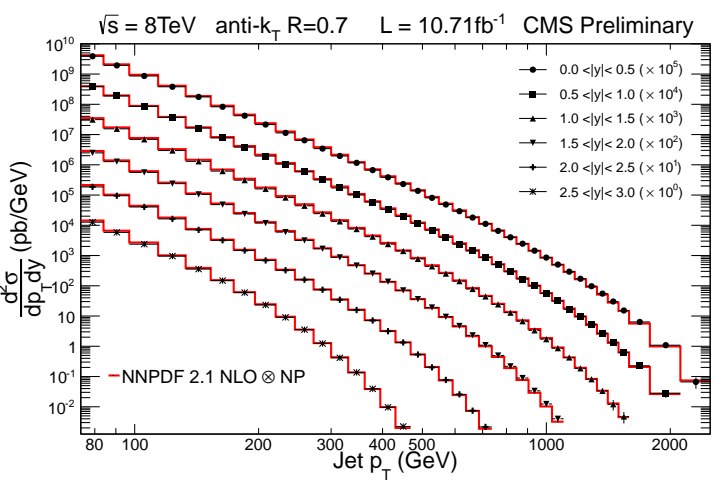

Figure 1. Double-differential inclusive jet cross section (points) in comparison to NLO predictions (red lines) using the NNPDF2.1 PDF set. Data points are shown for six rapidity ranges.

from PDF variation and ranges from 10-50\%. The measured cross section matches the theory calculation with the NNPDF2.1 PDF set [6], as shown in Fig. 1.

Data was compared to predictions from five PDF sets: ABM11 [7], CT10 [8], HERAPDF1.5 [9], MSTW2008NLO [10], and NNPDF2.1. All match data within the uncertainties except for ABM11 in certain rapidity ranges. These results provide strong confirmation for the NLOJet++ calculations and PDFs.

\section{Search for Jet Extinction in the Inclusive Jet $p_{T}$ Spectrum}

Terascale gravity theories predict that the Planck scale would occur at the $\mathrm{TeV}$ scale [11]. In this case, collisions at the LHC could produce microscopic black holes. Many such theories predict that these black holes would 


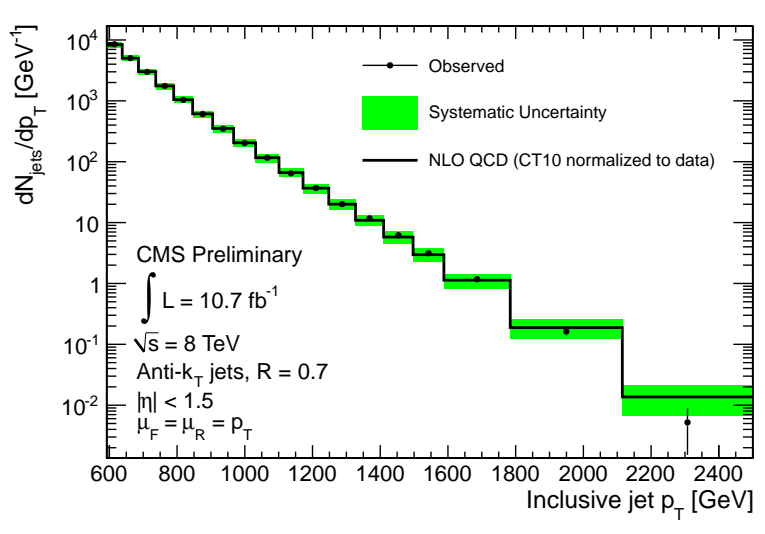

Figure 2. Inclusive jet $p_{T}$ spectrum (points) for $|\eta|<1.5$, as observed in $10.7 \mathrm{fb}^{-1}$ of data. The SM NLO simulation, convolved with the detector response and normalized to the total observed cross section, is shown by the solid line. The colored band shows the magnitude of all sources of systematic uncertainty added in quadrature.

decay spectacularly in a spray of high- $p_{T}$ jets and particles $[12,13]$, but LHC searches so far have found no evidence for such black hole decay [14-22]. However, nonperturbative processes could produce black holes that decay to a high multiplicity of low-energy jets [23]. Such black hole decays might be difficult to distinguish from the low-energy SM background, but their effect on the jet$p_{T}$ spectrum would be dramatic. At a certain energy scale, black holes would dominate and thereby suppress the production of high- $p_{T}$ jets, causing jet extinction at the high end of the jet- $p_{T}$ spectrum.

CMS has performed a search for such jet extinction with $10.7 \mathrm{fb}^{-1}$ of the $20128 \mathrm{TeV}$ pp dataset [24]. The SM prediction was made at NLO with NLOJet++ in the fastNLO framework and the CT10 PDF set and was scaled to data. The leading-order extinction spectrum was generated with PYTHIA [25] and assumed the strong-coupling limit of the string model, so jet extinction would occur beyond a scale $M$. The largest systematic uncertainty comes from the jet energy scale and is about $10 \%$. The data was found to match the SM prediction within the uncertainties, as seen in Figs. 2 and 3. A 95\% confidence level (CL) limit at $3.3 \mathrm{TeV}$ on scale $M$ was set with the $\mathrm{CL}_{S}$ calculator, as shown in Fig. 4.

For the NLOJet++ calculator, there are a number of PDF sets that can be employed, in addition to the CT10 PDF used for the SM estimate. The variation between PDF sets is bracketed by the CT10 and MSWT2008 sets. Thus, as a cross check, the MSWT2008 set was tried for the SM estimate, and it increased the observed limit by only $10 \%$, thereby confirming the CT10 limit of $3.3 \mathrm{TeV}$ as a conservative value.

\section{Search for Narrow Resonances using the Dijet Mass Spectrum}

Many new physics models predict heavy resonances that couple to quarks and gluons and decay to dijets. Some

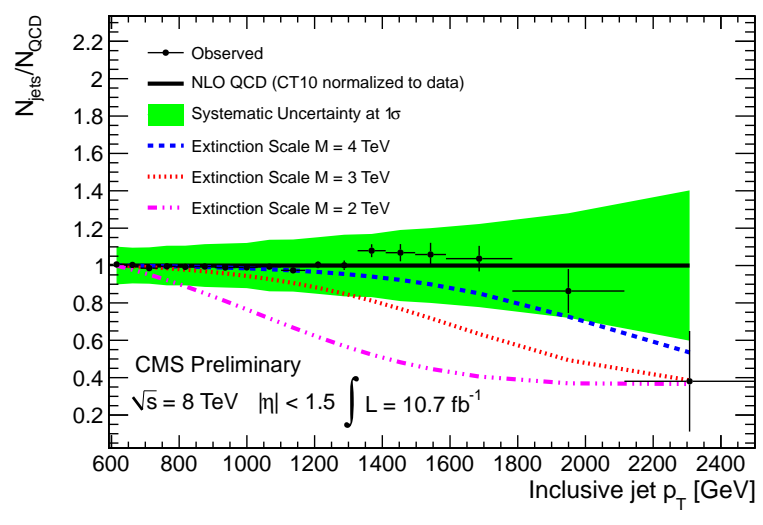

Figure 3. The ratio of the inclusive jet- $p_{T}$ spectrum to the NLO QCD prediction convolved with the detector resolution. The colored band shows the magnitude of all sources of systematic uncertainty added in quadrature at $1 \sigma$. Dashed lines indicate the effects of extinction at three different values of the extinction scale, $M=2,3$, and $4 \mathrm{TeV}$.

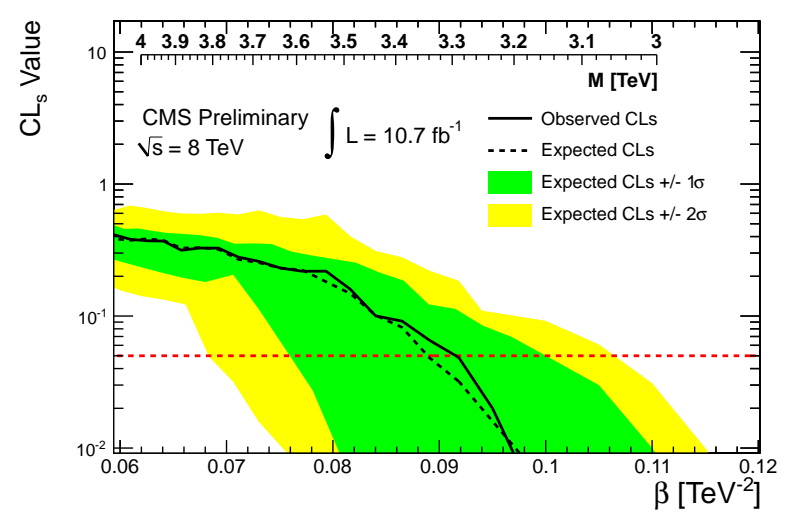

Figure 4. The results of a $\mathrm{CL}_{\mathrm{s}}$ scan in the extinction scale, $\beta=M^{-2}$. The observed value of $\mathrm{CL}_{\mathrm{s}}$ as a function of $\beta$ is shown by the solid line. The observed upper limit on $\beta$ is $0.091 \mathrm{TeV}^{-2}$ at $95 \% \mathrm{CL}$, corresponding to a lower limit of $3.3 \mathrm{TeV}$ on the extinction scale $M$. The dashed line indicates the expected median of results for the background-only hypothesis. The green (dark) and yellow (light) bands indicate the ranges that are expected to contain $68 \%$ and $95 \%$ of all observed excursions of the background from the median, respectively. The red line represents the $95 \%$ CL.

of these models include axigluons (A) [26, 27], coloroctet colorons (C) [28], excited quarks [29, 30], RandallSundrum gravitons [31], scalar diquarks [32], string resonances [33, 34], technicolor s8 resonances [35], and new gauge bosons (W' and Z') [36]. CMS has performed searches for such resonances, first with $4.0 \mathrm{fb}^{-1}$ [37], and then with the full $19.6 \mathrm{fb}^{-1} 20128 \mathrm{TeV}$ pp dataset [38]. This search employs the wide-jet technique [39-41], which adds close sub-leading jets to the two leading jets in each selected event. Figure 5 shows a CMS event display of the dijet event with the highest invariant mass in this dijet search. 

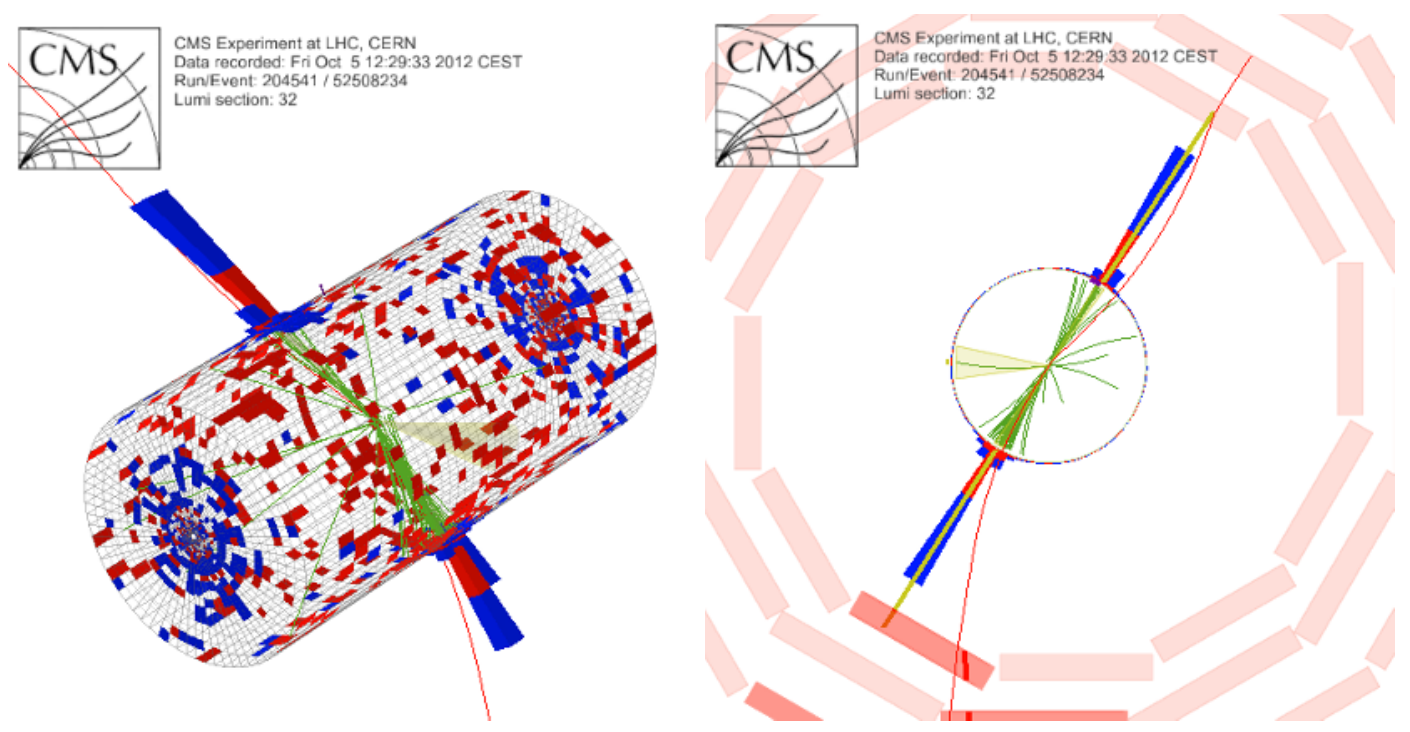

Figure 5. The event from the dijet search with the highest invariant mass: $3 \mathrm{D}$ view (left) and 2D view (right). The invariant mass of the two wide jets is $5.15 \mathrm{TeV}$.

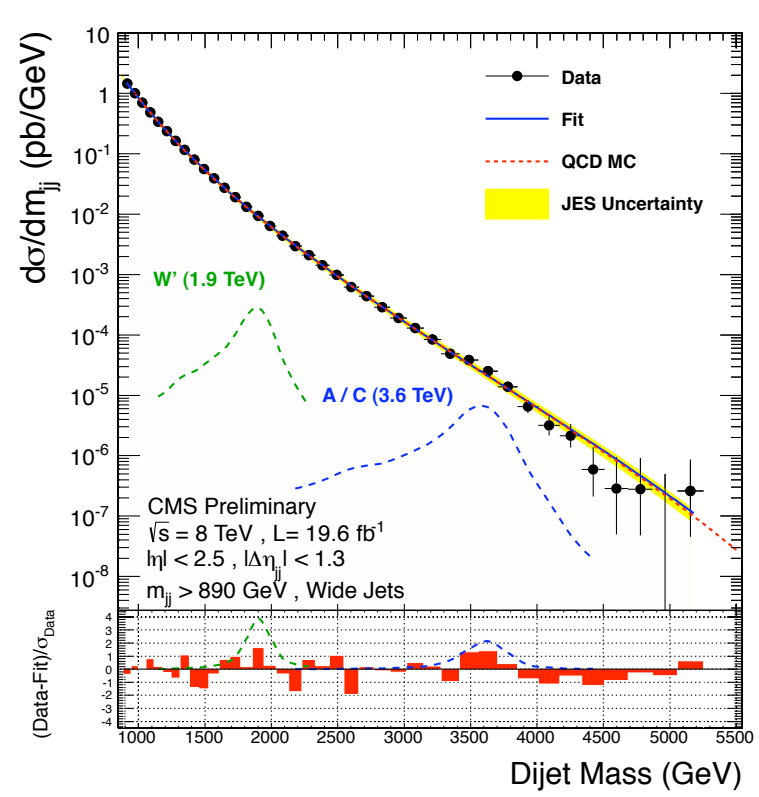

Figure 6. Dijet mass spectrum from wide jets (points) compared to a smooth fit (solid) and to predictions including detector simulation of QCD and signal resonances. The QCD prediction has been normalized to the data. The error bars are statistical only. The bin-by-bin fit residuals, (data-fit) $/ \sigma_{\text {data }}$, are shown at the bottom.

The background prediction comes from a fourparameter fit to the data. The largest systematic uncertainty is the jet energy resolution uncertainty, which is $10 \%$. The data matches the background estimate with no excess or bumps observed on the smooth background, as shown in Fig. 6. Mass limits are set on the eight signal

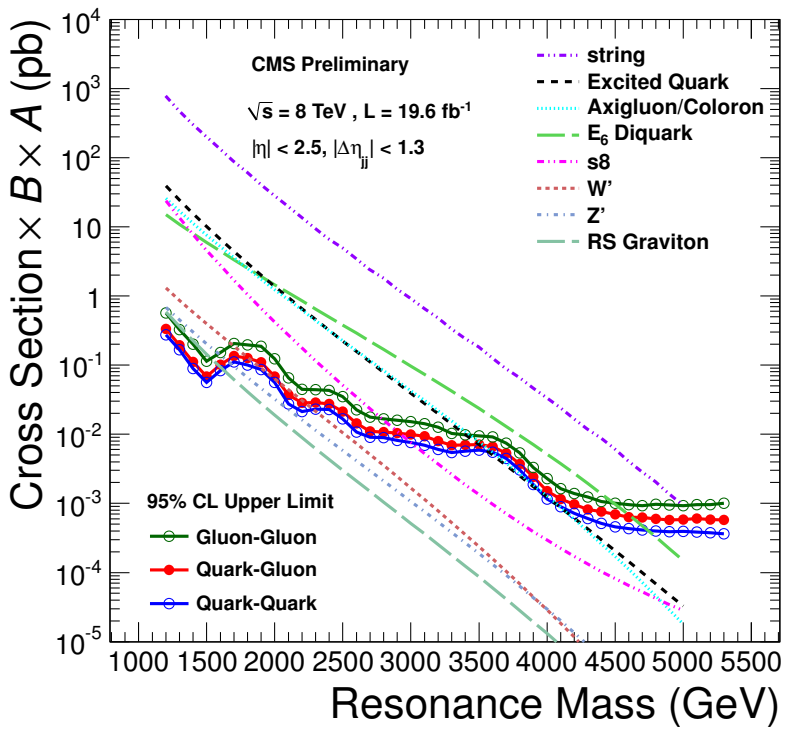

Figure 7. The observed $95 \%$ CL upper limits on $\sigma \times B \times A$ for dijet resonances of the type gluon-gluon, quark-gluon, and quarkquark, compared to theoretical predictions for string resonances, $\mathrm{E}_{6}$ diquarks, excited quarks, axigluons, colorons, s8 resonances, new gauge bosons $\mathrm{W}^{\prime}$ and $\mathrm{Z}^{\prime}$, and $\mathrm{RS}$ gravitons.

models, as shown in Fig. 7, with the strongest limit being $5.1 \mathrm{TeV}$ on the string resonance mass.

\section{Search for Heavy Resonances Decaying into $b \bar{b}$ and $b g$ Final States}

As a variation on the dijet search in Sec. 4, a b-jet tagging requirement can be placed on the jets in order to reduce SM backgrounds and to make the search sensi- 


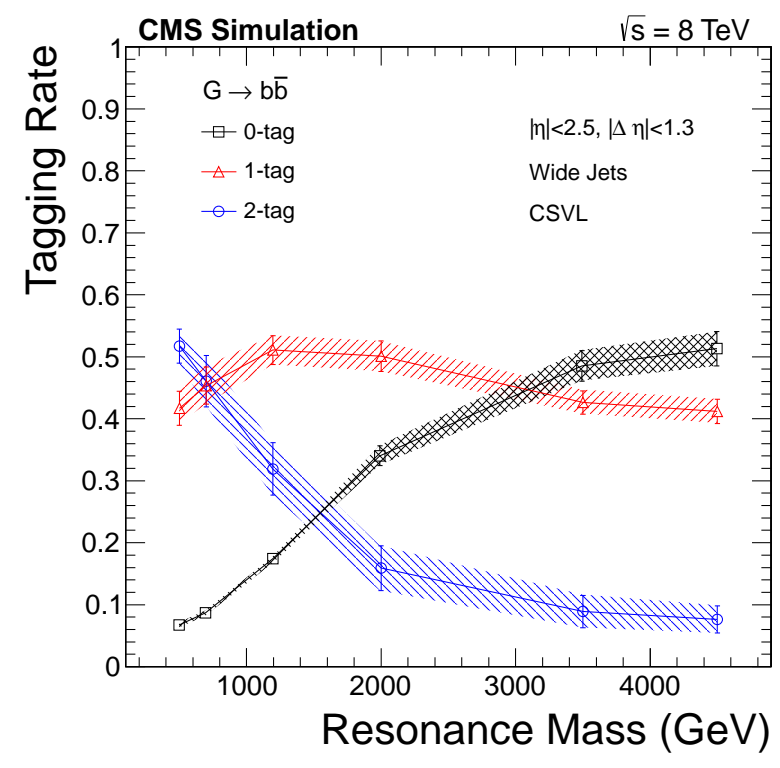

Figure 8. Tagging rates for 0,1 , and $2 \mathrm{~b}$ tags as a function of the resonance mass for the $b \overline{\mathbf{b}}$ decay mode. The hatched regions represent uncertainties in the tagging rates due to the variation of the b-tag scale factors within their uncertainties.

tive to models that specifically produce $b$ jets: excited $b$ quarks [29, 30], RS gravitons [31], and a sequential SM Z' [36]. CMS has performed such a search with the full $19.6 \mathrm{fb}^{-1} 20128 \mathrm{TeV}$ pp dataset [42]. Like the previous analysis, this one uses the wide-jet technique and a background estimate from a four-parameter fit to the data.

Because the b-tagging rate drops significantly for highmass signal resonances, as shown in Fig. 8, the analysis is performed in three channels: $0 \mathrm{~b}$ tags, $1 \mathrm{~b}$ tag, and $2 \mathrm{~b}$ tags.

The largest systematic uncertainty comes from the jet energy resolution and is about $10 \%$. The background estimates compared to the data are shown in Fig. 9. The data match the background estimates within the uncertainties, and no excess is observed. The best mass limits to date are set on the three signal models, as shown in Fig. 10, with the strongest limit being $1.7 \mathrm{TeV}$ on the $\mathrm{Z}$ ' mass.

\section{Conclusion}

The CMS Collaboration has measured the inclusive jet cross section and performed several searches for new physics using jets with the $20128 \mathrm{TeV}$ pp collision dataset. The jet cross section measurement has confirmed the NLOJet++ calculations and PDF sets. In the searches, no significant deviations from the SM were observed. New limits have been set on many models, most being the best to date on these models. These limits range up to $3.3 \mathrm{TeV}$ on the jet extinction scale, up to $5.1 \mathrm{TeV}$ on dijet string resonances, and up to $1.7 \mathrm{TeV}$ on a $\mathrm{Z}$ '. CMS is continuing its searches for new physics, and more results will be coming out soon.

\section{References}

[1] S. Chatrchyan et al. (CMS), JINST 3, S08004 (2008)

[2] S. Chatrchyan et al. (CMS), CMS Physics Analysis Summary CMS-PAS-SMP-12-012 (2013)

[3] Z. Nagy, Phys. Rev. D 68, 094002 (2003), hep-ph/0307268

[4] Z. Nagy, Phys. Rev. Lett. 88, 122003 (2002), hep-ph/0110315

[5] D. Britzger, K. Rabbertz, F. Stober, M. Wobisch, p. 217 (2012), arXiv: 1208.3641

[6] R.D. Ball et al., Nucl. Phys. B 838, 136 (2010)

[7] S. Alekhin, J. Blümlein, S. Moch, Phys. Rev. D 86, 054009 (2012), arXiv: 1202.2281

[8] H.L. Lai et al., Phys. Rev. D 82, 074024 (2010)

[9] H1 and ZEUS Collaborations, JHEP 01, 109 (2010)

[10] A.D. Martin et al., Eur. Phys. J. C 63, 189 (2009)

[11] T. Banks, W. Fischler (1999), hep-th/9906038

[12] S.B. Giddings, S.D. Thomas, Phys. Rev. D 65, 056010 (2002)

[13] G. Landsberg, S. Dimopoulos, Phys. Rev. Lett. 87, 161602 (2001)

[14] G. Aad et al. (ATLAS), Phys. Lett. B 709, 322 (2012)

[15] G. Aad et al. (ATLAS), Phys. Lett. B 710, 538 (2012)

[16] G. Aad et al. (ATLAS), Phys. Lett. B 712, 331 (2012)

[17] S. Chatrchyan et al. (CMS), JHEP 04, 061 (2012)

[18] S. Chatrchyan et al. (CMS), Phys. Rev. Lett. 108, 111801 (2012)

[19] S. Chatrchyan et al. (CMS), Phys. Lett. B 704, 123 (2011)

[20] S. Chatrchyan et al. (CMS), JHEP 05, 093 (2011)

[21] S. Chatrchyan et al. (CMS), Phys. Rev. Lett. 108, 261803 (2012)

[22] S. Chatrchyan et al. (CMS), JHEP 1209, 094 (2012), arXiv: 1206.5663

[23] C. Kilic, A. Lath, K. Rose, S. Thomas (2012), submitted to PRD, arXiv: 1207.3525

[24] S. Chatrchyan et al. (CMS), CMS Physics Analysis Summary CMS-PAS-EXO-12-051 (2013)

[25] T. Sjöstrand, S. Mrenna, P.Z. Skands, JHEP 05, 026 (2006)

[26] P.H. Frampton, S.L. Glashow, Phys.Lett. B190, 157 (1987)

[27] R.S. Chivukula, A. Farzinnia, E.H. Simmons, R. Foadi, Phys.Rev. D85, 054005 (2012), arXiv: 1111.7261

[28] E.H. Simmons, Phys.Rev. D55, 1678 (1997), hep-ph/9608269

[29] U. Baur, I. Hinchliffe, D. Zeppenfeld, Int. J. Mod. Phys. A 2, 1285 (1987)

[30] U. Baur, M. Spira, P.M. Zerwas, Phys. Rev. D 42, 815 (1990)

[31] L. Randall, R. Sundrum, Phys. Rev. Lett. 83, 4690 (1999), hep-th/9906064

[32] J.L. Hewett, T.G. Rizzo, Phys.Rept. 183, 193 (1989)

[33] L.A. Anchordoqui, H. Goldberg, D. Lust, S. Nawata, S. Stieberger et al., Phys.Rev.Lett. 101, 241803 

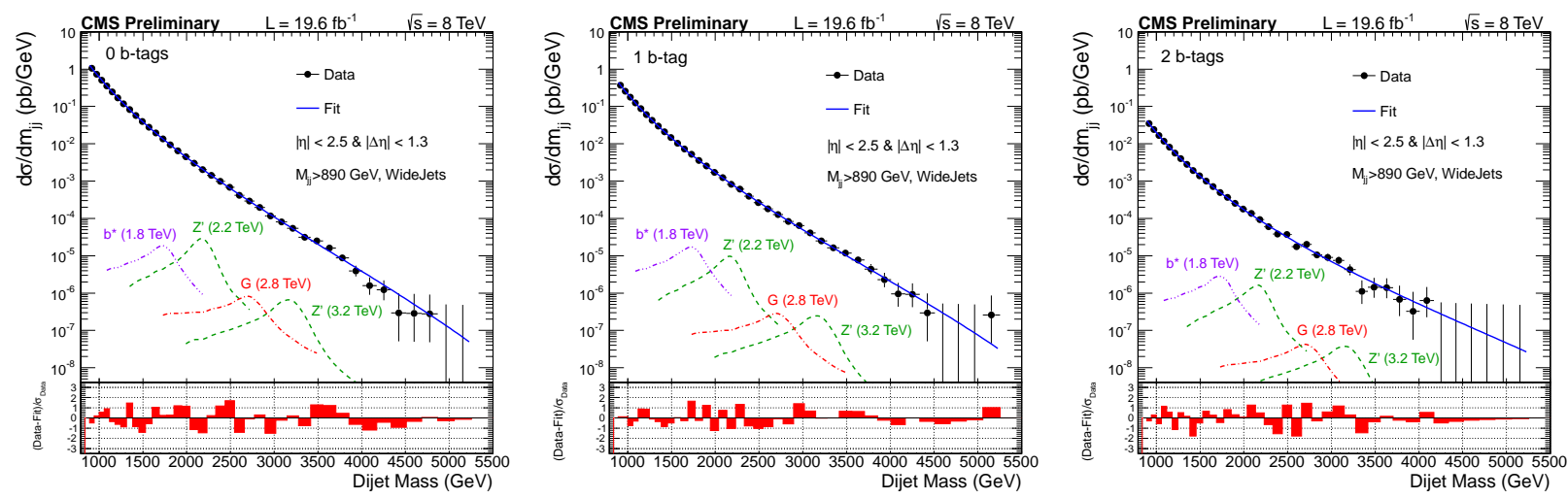

Figure 9. Dijet mass spectra (points) in different b-tag multiplicity bins compared to a smooth fit (solid line). The bin-by-bin fit residuals are shown at the bottom of each plot. Predictions for RS graviton, Z', and excited b-quark signal spectra are also shown. The vertical error bars are central intervals with correct coverage for Poisson variation, and the horizontal error bars are the bin widths.
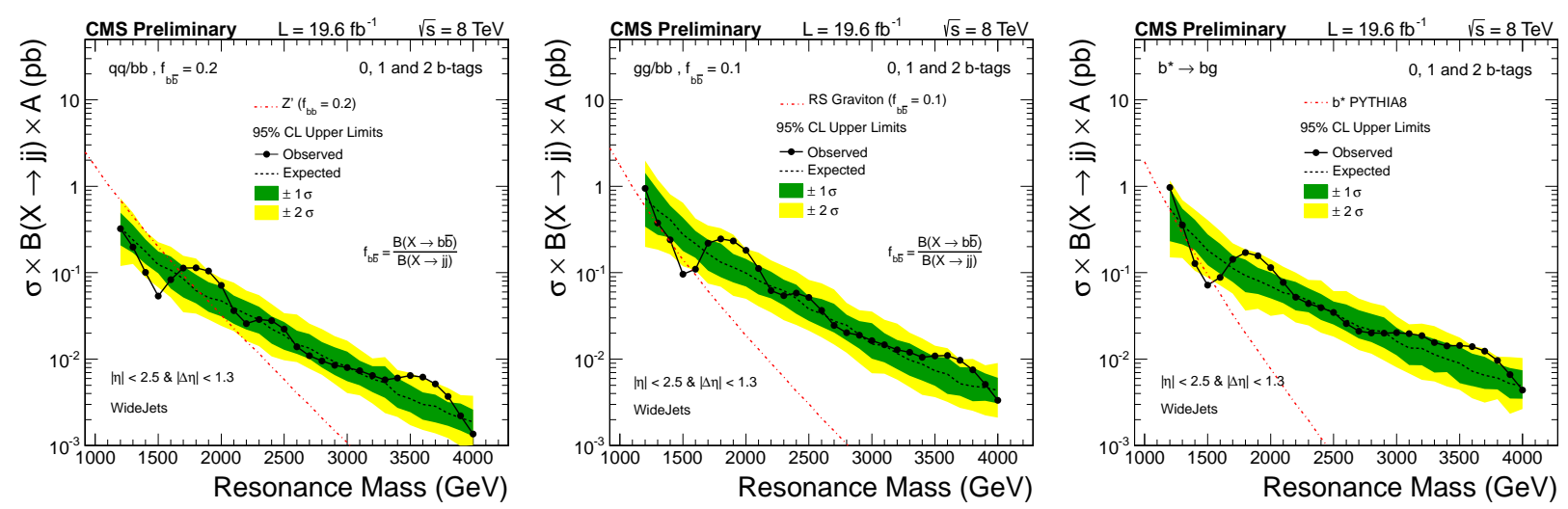

Figure 10. Combined observed and expected 95\% CL upper limits on $\sigma \times B \times A$ with systematic uncertainties included for qq/bb resonances with $f_{\mathrm{b} \overline{\mathrm{b}}}=0.2$ (left), gg/bb resonances with $f_{\mathrm{b} \overline{\mathrm{b}}}=0.1$ (middle), and $\mathrm{b}^{*} \rightarrow \mathrm{bg}$ resonances (right). $f_{\mathrm{b} \bar{b}}$ is the ratio of the branching fraction of the resonance decaying to $b \bar{b}$ over the resonance decaying to all jets. Theoretical cross sections for RS graviton, Z', and excited b quark are shown for comparison.

(2008), arXiv:0808.0497

[34] S. Cullen, M. Perelstein, M.E. Peskin, Phys.Rev. D62, 055012 (2000), hep-ph/0001166

[35] T. Han, I. Lewis, Z. Liu, JHEP 1012, 085 (2010), arXiv: 1010.4309

[36] E. Eichten, I. Hinchliffe, K.D. Lane, C. Quigg, Rev. Mod. Phys. 56, 579 (1984)

[37] S. Chatrchyan et al. (CMS), CMS Physics Analysis Summary CMS-PAS-EXO-12-016 (2012)
[38] S. Chatrchyan et al. (CMS), CMS Physics Analysis Summary CMS-PAS-EXO-12-059 (2013)

[39] M. Cacciari, J. Rojo, G.P. Salam, G. Soyez, JHEP 0812, 032 (2008), arXiv: 0810.1304

[40] D. Krohn, J. Thaler, L.T. Wang, JHEP 02, 084 (2010)

[41] A. Abdesselam et al., Eur. Phys. J. C 71, 1661 (2011)

[42] S. Chatrchyan et al. (CMS), CMS Physics Analysis Summary CMS-PAS-EXO-12-023 (2013) 